\title{
CORRECTION TO \\ "COMBINATORICS AND GEOMETRY OF POWER IDEALS": TWO COUNTEREXAMPLES FOR POWER IDEALS OF HYPERPLANE ARRANGEMENTS
}

\author{
FEDERICO ARDILA AND ALEXANDER POSTNIKOV
}

\begin{abstract}
We disprove Holtz and Ron's conjecture that the power ideal $C_{\mathcal{A},-2}$ of a hyperplane arrangement $\mathcal{A}$ (also called the internal zonotopal space) is generated by $\mathcal{A}$-monomials. We also show that, in contrast with the case $k \geq-2$, the Hilbert series of $C_{\mathcal{A}, k}$ is not determined by the matroid of $\mathcal{A}$ for $k \leq-6$.
\end{abstract}

Remark. This note is a corrigendum to our article [1], and we follow the notation of that paper.

\section{INTRODUCTION}

Let $\mathcal{A}=\left\{H_{1}, \ldots, H_{n}\right\}$ be a hyperplane arrangement in a vector space $V$; say $H_{i}=\left\{x \mid l_{i}(x)=0\right\}$ for some linear functions $l_{i} \in V^{*}$. Call a product of (possibly repeated) $l_{i}$ 's an $\mathcal{A}$-monomial in the symmetric algebra $\mathbb{C}\left[V^{*}\right]$. Let Lines $(\mathcal{A})$ be the set of lines of intersection of the hyperplanes in $\mathcal{A}$. For each $h \in V$ with $h \neq 0$, let $\rho_{\mathcal{A}}(h)$ be the number of hyperplanes in $\mathcal{A}$ not containing $h$. Let $\rho=\rho(\mathcal{A})=$ $\min _{h \in V}\left(\rho_{\mathcal{A}}(h)\right)$. For all integers $k \geq-(\rho+1)$, consider the power ideals

$$
I_{\mathcal{A}, k}:=\left\langle h^{\rho_{\mathcal{A}}(h)+k+1} \mid h \in V, h \neq 0\right\rangle, \quad I_{\mathcal{A}, k}^{\prime}:=\left\langle h^{\rho_{\mathcal{A}}(h)+k+1} \mid h \in \operatorname{Lines}(\mathcal{A})\right\rangle
$$

in the symmetric algebra $\mathbb{C}[V]$. It is convenient to regard the polynomials in $I_{\mathcal{A}, k}$ as differential operators, and to consider the space of solutions to the resulting system of differential equations:

$$
C_{\mathcal{A}, k}=I_{\mathcal{A}, k}^{\perp}:=\left\{f(x) \in \mathbb{C}\left[V^{*}\right] \mid h\left(\frac{\partial}{\partial x}\right)^{\rho_{\mathcal{A}}(h)+k+1} f(x)=0 \text { for all } h \neq 0\right\}
$$

which is known as the inverse system of $I_{\mathcal{A}, k}$. Define $C_{\mathcal{A}, k}^{\prime}$ similarly. These objects arise naturally in numerical analysis, algebra, geometry, and combinatorics. For references, see [1,3].

One important question is to compute the Hilbert series of these spaces of polynomials, graded by degree, as a function of combinatorial invariants of $\mathcal{A}$. Frequently, the answer is expressed in terms of the Tutte polynomial of $\mathcal{A}$. This has been done

Received by the editors November 7, 2012 and, in revised form, January 4, 2013.

2010 Mathematics Subject Classification. Primary 05A15, 05B35, 13P99, 41A15, 52C35.

The first author was supported in part by NSF Award DMS-0801075 and CAREER Award DMS-0956178.

The second author was supported in part by NSF CAREER Award DMS-0504629. 
successfully in many cases. One strategy used independently by different authors has been to prove the following:

(i) There is a spanning set of $\mathcal{A}$-monomials for $C_{\mathcal{A}, k}$.

(ii) There is an exact sequence $0 \rightarrow C_{\mathcal{A} \backslash H, k}(-1) \rightarrow C_{\mathcal{A}, k} \rightarrow C_{\mathcal{A} / H, k} \rightarrow 0$ of graded vector spaces.

(iii) Therefore, the Hilbert series of $C_{\mathcal{A}, k}$ is an evaluation of the Tutte polynomial of $\mathcal{A}$.

Here $\mathcal{A} \backslash H$ and $\mathcal{A} / H$ are the deletion and contraction of $H$, respectively.

For $k \geq-1$, this method works very nicely. Dahmen and Micchelli [2] were the first ones to do this for $C_{\mathcal{A},-1}^{\prime}$. Postnikov, Shapiro, and Shapiro [5] did it for $C_{\mathcal{A}, 0}$, while Holtz and Ron [3] did it for $C_{\mathcal{A}, 0}^{\prime}$. In [1] we did it for $C_{\mathcal{A}, k}$ for all $k \geq-1$, and showed that $C_{\mathcal{A}, 0}^{\prime}=C_{\mathcal{A}, 0}$ and $C_{\mathcal{A},-1}^{\prime}=C_{\mathcal{A},-1}$.

For $k \leq-3$ this approach does not work in full generality. In [1] we showed that (i) is false in general for $C_{\mathcal{A}, k}$, and left (ii) and (iii) open, suggesting the problem of measuring $C_{\mathcal{A}, k}$. For $k \leq-6$, (ii) and (iii) are false, as we will show in Propositions 4 and 5 . respectively. In fact, we will see that the Hilbert series of $C_{\mathcal{A}, k}$ is not even determined by the matroid of $\mathcal{A}$.

The intermediate cases are interesting and subtle, and deserve further study; notably the case $k=-2$, which Holtz and Ron call the internal zonotopal space. In [3] they proved (ii) and (iii) and conjectured (i) for $C_{\mathcal{A},-2}^{\prime}$. In [1, Proposition 4.5.3] - a restatement of Holtz and Ron's Conjecture 6.1 in [3] - we put forward an incorrect proof of this conjecture; the last sentence of our argument is false. In fact their conjecture is false, as we will see in Proposition 2 .

\section{The CASe $k=-2$ : Internal zonotopal spaCeS}

Before showing why Holtz and Ron's conjecture is false, let us point out that the remaining statements about $C_{\mathcal{A},-2}$ that we made in [1] are true. The easiest way to derive them is to prove that $C_{\mathcal{A},-2}=C_{\mathcal{A},-2}^{\prime}$, and simply note that Holtz and Ron already proved those statements for $C_{\mathcal{A},-2}^{\prime}$ :

Lemma 1. We have $C_{\mathcal{A}, k}=C_{\mathcal{A}, k}^{\prime}$ for any $k$ with $-(\rho+1) \leq k \leq 0$.

Proof. By [1, Theorem 4.17] we have $I_{\mathcal{A}, 0}=I_{\mathcal{A}, 0}^{\prime}$, so it suffices to show that $I_{\mathcal{A}, j}=I_{\mathcal{A}, j}^{\prime}$ implies that $I_{\mathcal{A}, j-1}=I_{\mathcal{A}, j-1}^{\prime}$ as long as these ideals are defined. If $I_{\mathcal{A}, j}=I_{\mathcal{A}, j}^{\prime}$, then for any $h \in V \backslash\{0\}$ we have $h^{\rho_{\mathcal{A}}(h)+j+1}=\sum f_{i} h_{i}^{\rho_{\mathcal{A}}\left(h_{i}\right)+j+1}$ for some polynomials $f_{i}$, where the $h_{i}$ 's are the lines of the arrangement. As long as the exponents are positive, taking partial derivatives in the direction of $h$ gives $h^{\rho_{\mathcal{A}}(h)+j}=\sum g_{i} h_{i}^{\rho_{\mathcal{A}}\left(h_{i}\right)+j}$ for some polynomials $g_{i}$.

The following result shows that (i) does not hold for $C_{\mathcal{A},-2}$.

Proposition 2. [3, Conjecture 6.1] is false: The "internal zonotopal space" $C_{\mathcal{A},-2}$ is not necessarily spanned by $\mathcal{A}$-monomials.

Proof. Let $\mathcal{H}$ be the hyperplane arrangement in $\mathbb{C}^{4}$ determined by the linear forms $y_{1}, y_{2}, y_{3}, y_{1}-y_{4}, y_{2}-y_{4}, y_{3}-y_{4}$. We have

$$
I_{\mathcal{H},-2}^{\prime}=\left\langle x_{1}^{1}, x_{2}^{1}, x_{3}^{1},\left(\epsilon_{1} x_{1}+\epsilon_{2} x_{2}+\epsilon_{3} x_{4}+x_{4}\right)^{2}\right\rangle=\left\langle x_{1}, x_{2}, x_{3}, x_{4}^{2}\right\rangle
$$


as $\epsilon_{1}, \epsilon_{2}, \epsilon_{3}$ range over $\{0,1\}$. The other generators of $I_{\mathcal{H},-2}$ are of degree at least 3 , and are therefore in $I_{\mathcal{H},-2}^{\prime}$ already, so

$$
I_{\mathcal{H},-2}=\left\langle x_{1}, x_{2}, x_{3}, x_{4}^{2}\right\rangle, \quad C_{\mathcal{H},-2}=\operatorname{span}\left(1, y_{4}\right) .
$$

Therefore $C_{\mathcal{H},-2}$ is not spanned by $\mathcal{H}$-monomials.

As Holtz and Ron pointed out, if [3, Conjecture 6.1] had been true, it would have implied [3, Conjecture 1.8], an interesting spline-theoretic interpretation of $C_{\mathcal{A},-2}$ when $\mathcal{A}$ is unimodular. The arrangement above is unimodular, but it does not provide a counterexample to [3, Conjecture 1.8]. In fact, Matthias Lenz [4] has recently put forward a proof of this weaker conjecture.

\section{THE CASE $k \leq-6$}

In this section we show that when $k \leq-6$, the Hilbert series of $C_{\mathcal{A}, k}$ is not a function of the Tutte polynomial of $\mathcal{A}$. In fact, it is not even determined by the matroid of $\mathcal{A}$. Recall that $\rho=\rho(\mathcal{A}):=\min _{h \in V}\left(\rho_{\mathcal{A}}(h)\right)$. Say $h \in V$ is large if it is on the maximum number of hyperplanes, so $\rho_{\mathcal{A}}(h)=\rho$.

Lemma 3. The degree 1 component of $C_{\mathcal{A},-\rho}$ is

$$
\left(C_{\mathcal{A},-\rho}\right)_{1}=(\operatorname{span}\{h \in V: h \text { is large }\})^{\perp}
$$

in $V^{*}$.

Proof. An element $f$ of $C_{\mathcal{A},-\rho}$ needs to satisfy the differential equations $h(\partial / \partial x)^{\rho_{\mathcal{A}}(h)-\rho+1} f(x)=0$ for all non-zero vectors $h \in V$. If $f$ is linear, then this condition is trivial unless $h$ is large; and in that case it says that $f \perp h$.

Proposition 4. For $k \leq-6$, the Hilbert series of $C_{\mathcal{A}, k}$ is not determined by the matroid of $\mathcal{A}$.

Proof. First assume $k=-2 m$. Let $L_{1}, L_{2}, L_{3}$ be three different lines through 0 in $\mathbb{C}^{3}$ and consider an arrangement $\mathcal{A}$ of $3 m$ (hyper)planes consisting of $m$ generically chosen planes $H_{i 1}, \ldots, H_{i m}$ passing through $L_{i}$ for $i=1,2,3$. Then $\rho=2 m$ and the only large lines are $L_{1}, L_{2}$, and $L_{3}$. Therefore $\operatorname{dim}\left(C_{\mathcal{A},-2 m}\right)_{1}$ equals 1 if $L_{1}, L_{2}, L_{3}$ are coplanar, and 0 otherwise. However, the matroid of $\mathcal{A}$ does not know whether $L_{1}, L_{2}, L_{3}$ are coplanar.

More precisely, consider two versions $\mathcal{A}_{1}$ and $\mathcal{A}_{2}$ of the above construction; in $\mathcal{A}_{1}$ the lines $L_{1}, L_{2}, L_{3}$ are coplanar, and in $\mathcal{A}_{2}$ they are not. Notice that $\mathcal{A}_{1}$ and $\mathcal{A}_{2}$ have the same matroid: the rank 3 matroid whose non-bases are the triples $\left\{H_{i a}, H_{i b}, H_{i c}\right\}$ for $1 \leq i \leq 3$ and $1 \leq a<b<c \leq m$. However, $\operatorname{dim}\left(C_{\mathcal{A}_{1},-2 m}\right)_{1} \neq$ $\operatorname{dim}\left(C_{\mathcal{A}_{2},-2 m}\right)_{1}$.

The case $k=-2 m-1$ is similar. It suffices to add a generic plane to the previous arrangements.

Proposition 5. For $k \leq-6$, the sequence of graded vector spaces

$$
0 \rightarrow C_{\mathcal{A} \backslash H, k}(-1) \rightarrow C_{\mathcal{A}, k} \rightarrow C_{\mathcal{A} / H, k} \rightarrow 0
$$

of [1, Proposition 4.4.1] is not necessarily exact, even if $H$ is neither a loop nor a coloop. 
Proof. We will not need to recall the maps that define this sequence; we will simply show an example where right exactness is impossible because $\operatorname{dim}\left(C_{\mathcal{A}, k}\right)_{1}=0$ and $\operatorname{dim}\left(C_{\mathcal{A} / H, k}\right)_{1}=1$. We do this in the case $k=-2 m$; the other one is similar.

Consider the arrangement $\mathcal{A}=\mathcal{A}_{2}$ of the proof of Proposition 4 and the plane $H=H_{11}$. We have $\operatorname{dim}\left(C_{\mathcal{A},-2 m}\right)_{1}=0$. In the contraction $\mathcal{A} / H$, the planes $H_{12}, \ldots, H_{1 m}$ become the same line $L_{1}$ in $H$, while the other $2 m$ planes of $\mathcal{A}$ become generic lines in $H$. Therefore $\rho(\mathcal{A} \backslash H)=2 m$ and $\left(C_{\mathcal{A} / H,-2 m}\right)_{1}=L_{1}^{\perp}$ in $H^{*}$, which is one-dimensional.

\section{ACKNOWLEDGMENTS}

The authors are very thankful to Matthias Lenz for pointing out the error in [1, and to Andrew Berget and Amos Ron for their comments on a preliminary version of this note. We also thank the referee for a suggestion which improved the exposition.

\section{REFERENCES}

[1] Federico Ardila and Alexander Postnikov, Combinatorics and geometry of power ideals, Trans. Amer. Math. Soc. 362 (2010), no. 8, 4357-4384, DOI 10.1090/S0002-9947-10-05018-X. MR2608410 (2011g:05322)

[2] Wolfgang Dahmen and Charles A. Micchelli, On the local linear independence of translates of a box spline, Studia Math. 82 (1985), no. 3, 243-263. MR825481 (87k:41008)

[3] Olga Holtz and Amos Ron, Zonotopal algebra, Adv. Math. 227 (2011), no. 2, 847-894, DOI 10.1016/j.aim.2011.02.012. MR2793025(2012e:52065)

[4] Matthias Lenz, Interpolation, box splines, and lattice points in zonotopes, Preprint, 2012.

[5] Alexander Postnikov, Boris Shapiro, and Mikhail Shapiro, Algebras of curvature forms on homogeneous manifolds, Differential topology, infinite-dimensional Lie algebras, and applications, Amer. Math. Soc. Transl. Ser. 2, vol. 194, Amer. Math. Soc., Providence, RI, 1999, pp. 227-235. MR1729365(2001a:53079)

Department of Mathematics, San Francisco State University, 1600 Holloway Avenue, SAN Francisco, CALIFornia 94110

E-mail address: federico@sfsu.edu

Department of Mathematics, Massachusetts Institute of Technology, 77 MassachuSetts Avenue, Cambridge, Massachusetts 02139

E-mail address: apost@math.mit.edu 